\title{
Verification of the Indoor GPS System, by Comparison with Calibrated Coordinates and by Angular Reference
}

\author{
J E Muelaner, Z Wang, O Martin, J Jamshidi, P G Maropoulos \\ The University of Bath, Bath, BA2 7AY, UK \\ +44 (0) 7743845124 \\ +44 (0) 1225386928 \\ J.E.Muelaner@bath.ac.uk \\ www.muelaner.com/research
}

\begin{abstract}
This paper details work carried out to verify the dimensional measurement performance of the Indoor GPS (iGPS) system; a network of Rotary-Laser Automatic Theodolites (R-LATs). Initially tests were carried out to determine the angular uncertainties on an individual R-LAT transmitter-receiver pair. A method is presented of determining the uncertainty of dimensional measurement for a three dimensional coordinate measurement machine. An experimental procedure was developed to compare three dimensional coordinate measurements with calibrated reference points. The reference standard used to calibrate these reference points was a fringe counting interferometer with the multilateration technique employed to establish three dimensional coordinates. This is an extension of the established technique of comparing measured lengths with calibrated lengths. The method was found to be practical and able to establish that the expanded uncertainty of the basic iGPS system was approximately $1 \mathrm{~mm}$ at a $95 \%$ confidence level. Further tests carried out on a highly optimized version of the iGPS system have shown that the coordinate uncertainty can be reduced to $0.25 \mathrm{~mm}$ at a $95 \%$ confidence level.
\end{abstract}

Keywords: Coordinate Uncertainty, CMM, iGPS, Indoor GPS, Spatial Analyzer, USMN

This is an expanded version of the paper originally presented at the 6th International Conference on Digital Enterprise Technology in Hong Kong 14-16 December 2009 and titled "VERIFICATION OF THE INDOOR GPS SYSTEM BY COMPARISON WITH POINTS CALIBRATED USING A NETWORK OF LASER TRACKER MEASUREMENTS"

iGPS: indoor GPS; R-LAT: Rotary-Laser Automatic Theodolite; MCS: Monte-Carlo Simulation; NPL: The National Physical Laboratory 


\section{Introduction}

Accurate measurement is of central importance to the manufacture of high quality products. In traditional manufacturing the master part definition existed as a physical reference model. A rational system of jigs, fixtures and gauges therefore embodied a direct system of traceability back to this master part. In modern engineering the master part is represented digitally as a mathematically precise $3 \mathrm{D}$ geometric model. The scale of the model is defined according to internationally agreed length standards based on the wavelength of light, with traceability back to time standards. In order to ensure part conformance, measurements should be taken using instruments which have been calibrated so as to ensure traceability back to these length standards as maintained by National Measurement Institutes such as the National Physical Laboratory.

Modern dimensional measurement systems do not simply measure lengths as is the case with traditional instruments such as micrometers and height gauges. Current industrial systems typically measure the three dimensional position of points on objects and therefore verification by the measurement of lengths cannot ensure the traceability of all coordinate measurements made by the instrument (Flack 2001).

The system verified in this work is a large volume frameless and distributed coordinate measurement machine that is made up of a network of Rotary-Laser Automatic Theodolites (R-LATs), this system is known commercially as Indoor GPS (iGPS). Each R-LAT consists of a transmitter and a sensor. The transmitter utilizes a rotating head to sweep two fanned lasers through the measurement volume; the transmitter also houses a strobe which fires a timing signal covering 360 degrees of azimuth, as shown in Figure 1. The sensor is able to detect the incidence of these lasers and deduce the azimuth and elevation angle from the transmitter to the sensor from the time differences between the strobe and the two lasers reaching the sensor (Hedges et al. 2003 ; Muelaner et al. 2008).

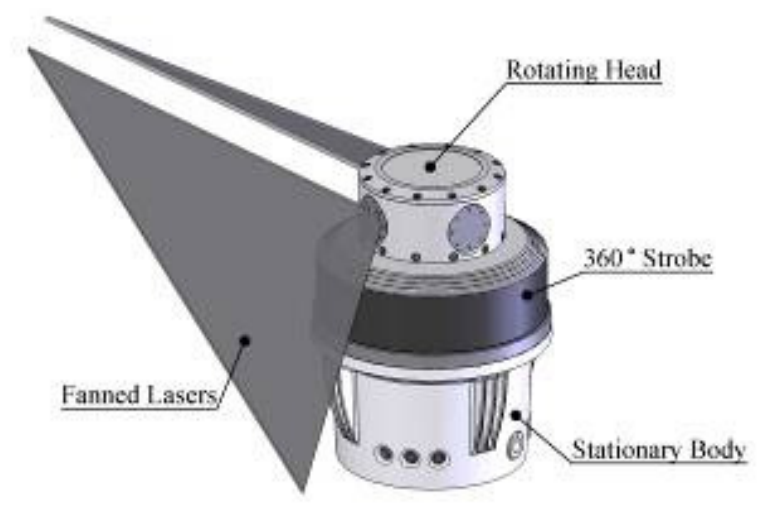

Figure 1: Main Components of R-LAT Transmitter

In order to detect the three dimensional coordinates of the sensor it must receive optical signals from at least two transmitters. It is then possible to use triangulation to fix the position of the sensor assuming that the transmitter positions are known. Normally, a bundle adjustment (Triggs et al. 1999) would be carried out as part of the setup procedure for the network. The bundle adjustment is used to establish the relative positions of the transmitters. If more than two transmitters are visible then some form of least squares fitting can be employed to reduce the uncertainty of the position.

Once the transmitter positions have been determined, the network of R-LATs then constitutes a large volume frameless coordinate measurement machine. This type of 
measurement network has advantages such as one way communication, from the transmitter network to the sensors, to support a virtually unlimited number of sensors. Additionally, a sensor is able to move behind line of sight obstructions, loosing and regaining connection to various transmitters without loosing connection to the network as a whole and not requiring any re-aiming of transmitters.

Typically, measurements are taken using a 'vector bar' shown in Figure 2. This is a calibrated device housing two sensors and with a 1.5" diameter precision sphere mounted at one end. The sensors and the sphere are mounted on a common axis. Since the position of the sensors can be calculated, the position of the vector bar is also known and the rotation can also be calculated in two axes. The position of the sphere is therefore known enabling it to be used as a measurement probe.

The advantage of this approach over typical measurement systems is that communication from the transmitter infrastructure to the sensors probes is one-way. This means that there is no practical limit to the scalability of the system. Additional transmitters can be added to the network in increase range and accuracy within a factory wide measurement volume. Since all communication is one-way; from the transmitters to the sensors; a practically unlimited number of sensors can be used within the transmitter infrastructure. These properties are very similar to the NAVSTAR GPS system which is used by millions of consumers with in-car navigation systems without increasing the infrastructure requirements.

In addition to more conventional product verification the highly scalable factory-wide measurement capabilities of the iGPS system might prove useful in applications such as the guidance of automation systems (Kapanoglu et al.), positioning of localized sensors within a wireless sensor network (Tang et al.) and delivery of location specific information to assembly or maintenance workers (Joshi et al. 2009).

\section{Verification strategy}

The body of literature concerning the verification of coordinate measurements is primarily concerned with comparison with calibrated lengths. The ISO 10360 standard for coordinate measuring machines (BSI - British Standards Institution 2002) is a well established work applicable to conventional gantry based coordinate measurement machines (CMMs) using contact probing and operating in the discrete-point probing mode. The ASME standard for evaluating 'Laser-Based Spherical Coordinate Measurement Systems' (ASME 2006), applicable to Laser Trackers (Lau et al. 1985) and Laser Radars (Richard and Kendall 2006) and the VDI/VDE standard for 'Optical 3D measuring systems' (VDI/VDE-Gesellschaft Mess- und Automatisierungstechnik 2002) are the only standards dealing with large volume frameless metrology instruments.

Both of the above standards are based on a methodology of measuring calibrated lengths at various orientations in order to test the isolated and combined accuracy of the instruments' sub-systems. Such sub-systems are the probing error and $\mathrm{x}, \mathrm{y}, \mathrm{z}$ encoders on a CMM, while on a laser tracker they are the two angle encoders, the interferometer and the probing error of the retro-reflector.

Applying the principle of isolating sub-systems initial work was carried out to characterize the performance of an individual R-LAT. This involved Monte-Carlo Simulation (MCS) and experimental calibration (Muelaner, Wang et al. 2008). Angular sub-system tests were followed by coordinate measurement tests of the complete iGPS system. This could have been carried out using the established method of measuring calibrated lengths, however, since this would not ensure traceability of coordinate measurements made by the system it was decided to develop a method based on three dimensional coordinates. 
The direct comparison of coordinates is not new, such an approach has, for example, been carried out to compare points measured on a surface with a laser scanner to points measured on the same surface with a conventional gantry type CMM (Anchini et al. 2007). However, such an approach does not give traceability since the coordinate measurements made by the CMM do not have direct tractability to a length standard.

A tracking interferometer has been used to measure the distance to a CMM head from multiple positions. These distances were then used to calculate coordinates using multilateration. Multilateration is a technique of combining multiple one-dimensional measurements to give three-dimensional measurements. It is therefore similar to the more widely known technique of triangulation but while triangulation combines multiple angular measurements, multilateration combines multiple length measurements. The difference between the nominal and the measured coordinates was then used to create an error map (Schwenke et al. 2005). The work described here follows essentially the same method with a few notable exceptions; standard industrial instruments are used such as a laser tracker and the measurements are used for verification by an assessment of measurement uncertainty (BSI 1995) rather than for error mapping.

The approach employed in this work involves the use of kinematic nests, shown in Figure 2 , to allow the repeatable positioning of both the reference measurement system and the system undergoing verification. These nests are commonly used to position the spherically mounted retro-reflectors (SMRs) used by laser trackers. Although the use of such nests will introduce additional uncertainty, this can be shown to be relatively small and quantifiable through repeated measurement with the reference system.

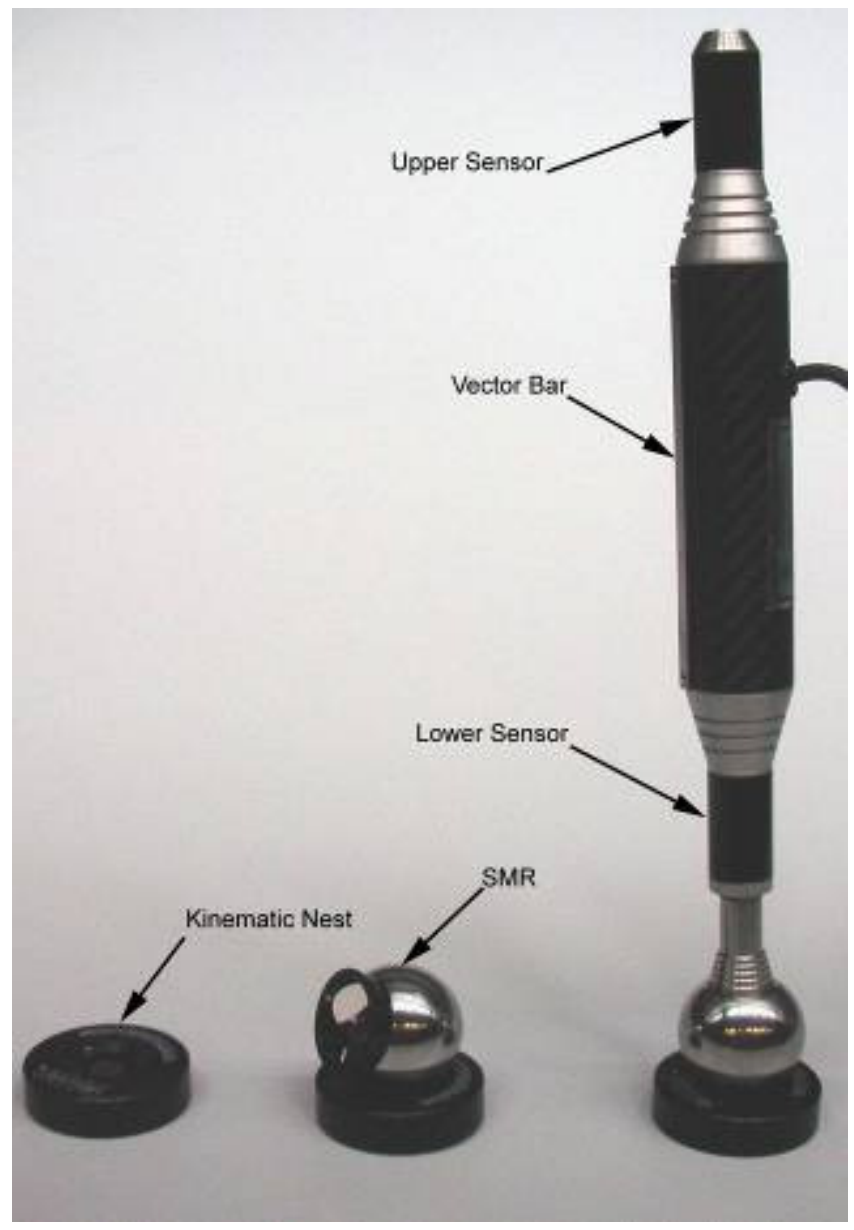

Figure 2 : Kinematic Nests with SMR and Vector Bar 


\section{Testing of Angular Sub-System}

Initial tests were carried out to evaluate the performance of an individual transmitterreceiver pair functioning as an automatic theodolite.

\section{Experimental Procedure}

The experimental procedure for angular sub-system tests involved comparing the azimuth measurement from the R-LAT with a reference angle established by a high precision rotary table. The transmitter was placed on the rotary table with the sensor located some distance away on a stable mounting. The azimuth angle was measured by the R-LAT, the transmitter rotated through a known reference angle using the rotary table and the new azimuth angle measured by the R-LAT. Finally the difference between the two azimuth readings from the R-LAT were compared with the reference angle (Muelaner, Wang et al. 2008).

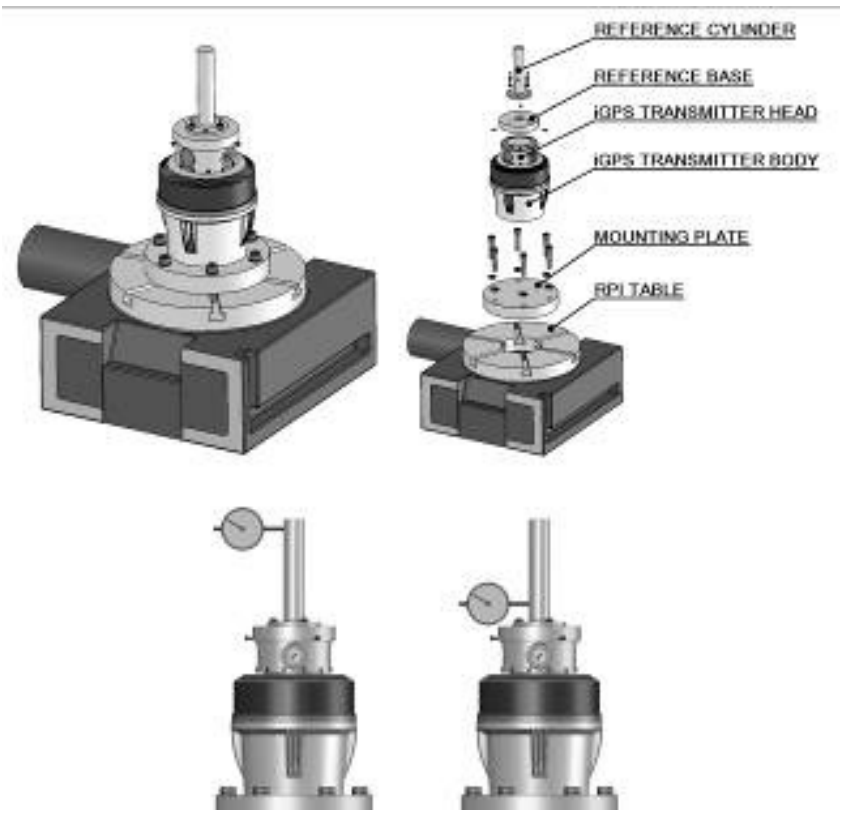

Figure 3: Assembly of Transmitter on Rotary Table

A critical factor in the setup of the reference angle was ensuring that the axis of rotation of the transmitter was coaxial with the rotation of the rotary table. A specially constructed reference cylinder was attached to the rotating head of the transmitter, as shown in Figure 3 , in such a way that it could be moved around on the surface of the centring base and also jacked-off the centring base so as to deviate slightly from the perpendicular in any direction. A centring procedure was carried out in two stages; the first stage was to rotate the R-LAT transmitter head adjusting the reference cylinder until it was co-axial with the transmitter's axis of rotation; the second stage was to rotate the rotary table adjusting the position transmitter until the reference cylinder was concentric with the table's axis of rotation. At both stages a dial gauge was used to check for deviations from coaxiality.

Evaluation of the uncertainty in the elevation angle was deemed impractical since:-

1. Results of tests for the azimuth measurement capability showed that the R-LAT probably has an uncertainty of equal to or better than the uncertainty in the reference angle, while simulations showed that the uncertainty in elevation angle must be lower than that in azimuth. A better reference standard would therefore be required to find any new information and such a reference standard was not available.

2. It would not be possible to simply rotate the transmitter through a known reference angle since changes in elevation angle might also move the position of the rotating head within its bearings invalidating the experiment. 
3. The alternative to rotating the transmitter through a known reference angle would be to move the sensor through a known angle. Since this rotation would be taking place at some considerable range the only feasible way to construct such a reference angle would be through the measurement of three lengths to construct a triangle. These lengths would consist of the distance between the upper and lower sensor position and the ranges from the origin of the transmitter's internal coordinate system to the two sensor positions. Unfortunately the exact position of the origin of the origin is not known. It is only known to be somewhere on the axis of rotation of the head.

\section{Results of Angular Tests}

The results of angular tests, presented previously (Muelaner, Wang et al. 2008), showed that uncertainty decreases with sampling duration, levelling off after 1 to 2 seconds. Other significant findings are that the uncertainty is relatively constant throughout the working volume and that the uncertainty value is not dependent on the size of the reference angle. The uncertainty in the azimuth angle measurement was approximately 0.5 arc seconds at a $95 \%$ confidence level, from basic trigonometry this is equivalent to 0.012 to $0.048 \mathrm{~mm}$ within the $5-20 \mathrm{~m}$ range.

\section{Experimental Procedure for Initial Coordinate Tests}

Two tests were carried out at different locations, both of which represented typical production environments. Although there were some differences between the tests the basic procedure was the same. The actual setup used for the tests carried out at the Bath Laboratory for Integrated Metrology Applications (LIMA) is shown in Figure 4.

\section{R-LAT Network Setup}

The R-LAT network was setup using the supplied interface software (Metris 2007) according to the user manual (Metris 2007). This involved positioning and starting the transmitters, setting various parameters and then connecting a vector bar to the network. The network consisted of 4 transmitters.

A bundle adjustment was carried out as specified in the user manual (Metris 2007). This involved taking measurements using the vector bar at 8 observation points within the working volume. The bundle was initially calculated using the known distance between the sensors on the vector bar to apply scale. Accurate lengths between two kinematic nests were then calibrated using an interferometer and the scale was reapplied by taking measurements of these nests with the vector bar.

\section{Coordinate Network Calibration}

The reference coordinates were created using kinematic nests designed to accept a 1.5" steel ball. A number of nests were glued to the concrete slab forming the floor of the test venue while others were mounted on either a granite table or theodolite stands.

Following the initial layout the coordinates of each kinematic nest were measured using a Laser Tracker. Measurements were taken from a number of positions allowing the results to be combined to improve accuracy using a technique similar to multilateration which is explained in section 0 . The number of positions differed between the tests. 


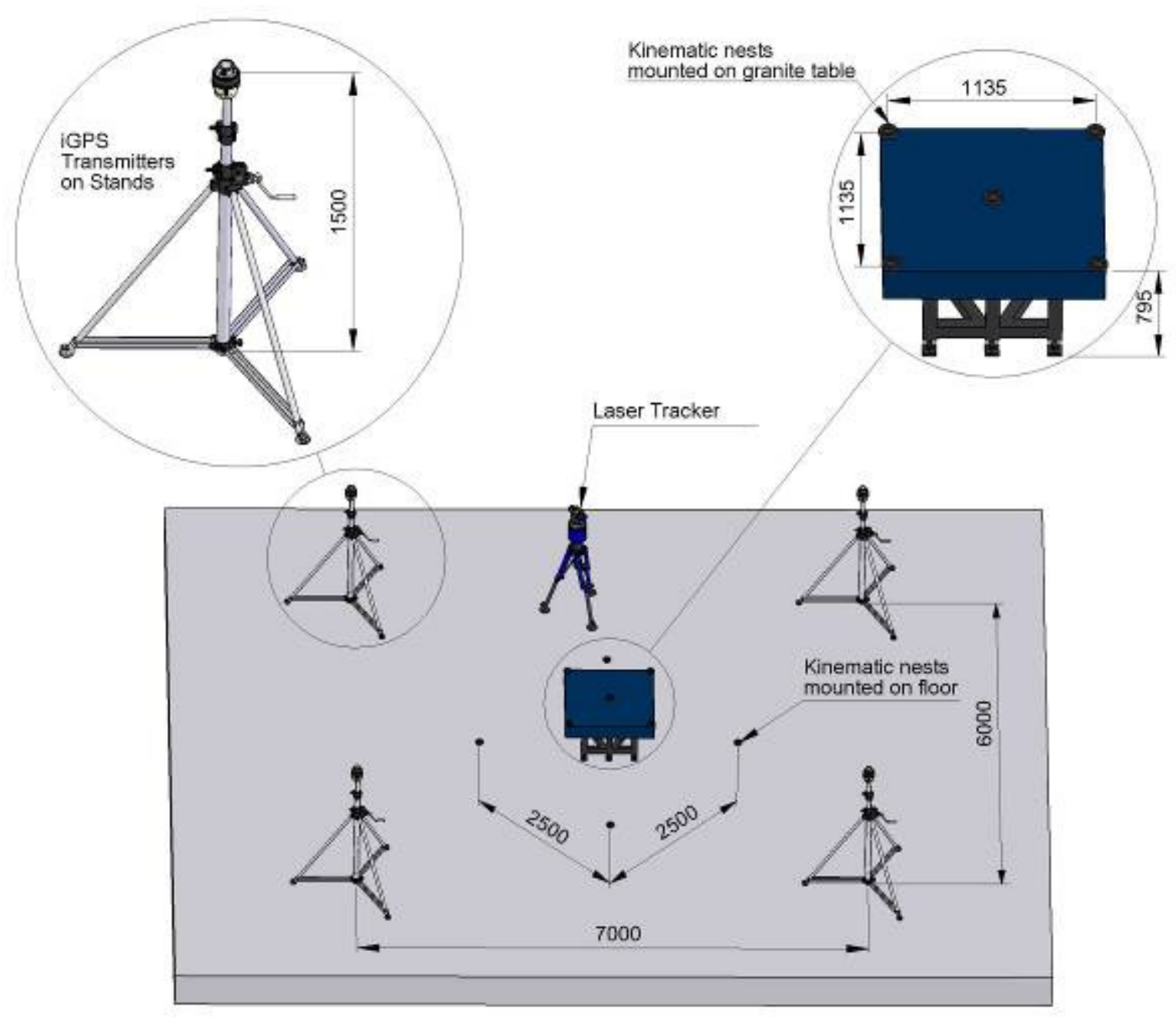

Figure 4: Test Setup for Tests at the Bath LIMA

\section{Replicated Measurements}

Following the coordinate network calibration the iGPS Vector Bar was used to make repeated measurements of the position of each kinematic nest. A 1.5" probe tip was used which was the same size as the SMR used for the Laser Tracker calibration. The points measured by the two methods are therefore equivalent. Each point was measured in turn using the Vector Bar and the measurements were then repeated a number of times measuring all the points in a circuit. The number of measurements of each point differed between the tests.

The system has a sampling frequency of approximately $40 \mathrm{~Hz}$ resulting from the rotational velocity of the transmitter heads. Due to the substantial effects of environmental disturbances such as turbulence on optical measurements (Estler et al. 2002) more accurate measurements can be made by averaging over a period of time. A single measurement of a coordinate position was therefore considered to be an average of 80 instantaneous measurements, this was regarded as giving a good compromise between accuracy and operation time (Muelaner, Wang et al. 2008).

An interface program was created to automate the measurement process and export of text files for further analysis. This interface software used rotation data from the Vector Bar to ensure that the Vector Bar was orientated vertically to within \pm 2 Degrees. The graphical user interface is shown in Figure 5. 


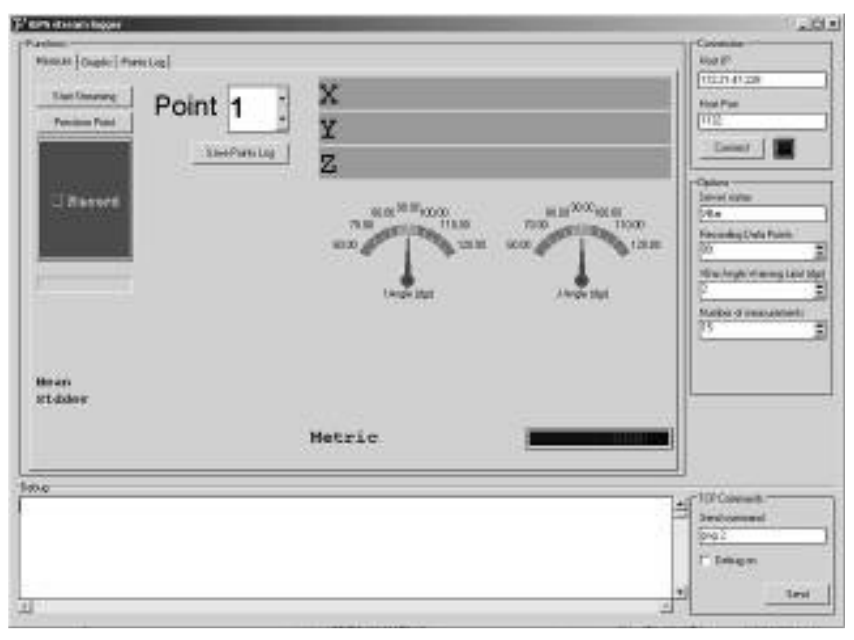

Figure 5 : Interface Software used to Collect Measurement Data

\section{Individual Experiments}

Although both tests used the experimental procedure detailed above there were some differences in the details of the setup and calibration. These differences are detailed in Table 1. The SMR nests for tests carried out at Bath's LIMA were located on the floor and a granite table. For the tests carried out at a large aircraft assembly area at Airbus Broughton, the nests were located on the floor and on theodolite stands.

Table 1 : Details of Individual Experiments

\begin{tabular}{|c|c|c|}
\hline Location & $\begin{array}{c}\text { Bath } \\
\text { LIMA }\end{array}$ & $\begin{array}{c}\text { Airbus } \\
\text { Broughton }\end{array}$ \\
\hline Date & $27 / 2 / 08$ & $4 / 3 / 08$ \\
\hline Laser Tracker Positions & 2 & 5 \\
\hline No. of Points & 9 & 15 \\
\hline Measurements per Point & 25 & 6 \\
\hline Transmitter Layout & $\begin{array}{c}9 \mathrm{~m} \times 7 \mathrm{~m} \\
\text { rectangle }\end{array}$ & $\begin{array}{c}12 \mathrm{~m} \mathrm{x} 12 \mathrm{~m} \\
\text { square }\end{array}$ \\
\hline $\begin{array}{c}\text { Scale Lengths } \\
\text { Used to Bundle }\end{array}$ & $5.6 \mathrm{~m}$ & $\begin{array}{c}8.3 \mathrm{~m}, 8.9 \mathrm{~m} \\
9.4 \mathrm{~m}, 11.2 \mathrm{~m}\end{array}$ \\
\hline
\end{tabular}

\section{Analysis of Results}

The analysis of results consisted of two stages. Firstly the calibration measurements of the coordinate network from multiple Laser Tracker positions were combined to obtain a reduced uncertainty for the point positions. The actual iGPS measurements were then analysed to calculate the uncertainty of the system.

\section{Coordinate Network Calibration}

The measurements from multiple laser tracker positions were combined into a single survey of the coordinate network using a technique which produces results similar to multilateration, reducing the coordinate uncertainty. This was achieved using a commercial code, Unified Spatial Metrology Network (USMN) which runs in the Spatial Analyzer (SA) software produced by New River Kinematics (NRK). This combines Monte Carlo analysis with best fitting of point clouds (Calkins 2002 ; New River Kinematics 2007).

The fundamentals of this technique are that the uncertainty of a particular measurement is simulated using knowledge of the position of the measurement instrument and the nonisotropic uncertainty of the instrument. The simulation is of the Monte Carlo type with 
repeated simulated measurements made, each consisting of the nominal measurement value with random noise added to it. In this way a point cloud or 'uncertainty field' of simulated measurements is created around each nominal measurement point. This uncertainty field can then be used to calculate the standard deviation of the coordinate measurement in each axis.

Each series of measurements of all the points from a single measurement station represents one point group. All of the point groups can then be best fitted to each other using a least squares minimization algorithm. The best fitting is weighted according to the uncertainty of each measurement. In this way points with, for example, a large standard deviation in the z-direction are allowed to deviate more in the z-direction from fitting to the corresponding points. The point groups are best fit to one another for each measurement in the uncertainty field in turn creating a new composite uncertainty field of the weighted best fits.

The repeated best fitting to generate a composite uncertainty field represents a second level of Monte Carlo simulation which is used to find the combined uncertainty for the coordinate measurements from multiple stations. Since the uncertainty of measurements taken using a laser tracker is known to be considerably better in range than in angle (Faro 2007), the distance measurements will be given greater weight than the angle derived measurements. The end result of this approach is therefore similar to multilateration. It is not however pure multilateration since the angle derived measurements are still used to some extent.

\section{Analysis of iGPS Measurements}

The mean of the replicated measurements of each point was calculated and these averaged measurements were best fitted to the calibrated positions (reference standard) using a least squares minimization algorithm. The distance between the mean position as measured using the iGPS network and the calibrated point position after best fitting all the points was then calculated, this uncorrected systematic error appeared to vary randomly throughout the measurement volume.

The uncertainty evaluation for the iGPS system is based on a comparison with the reference standard as established using a Laser Tracker and USMN as explained above. The uncertainty in this reference standard also includes the drift nest repeatability and the SMR centring tolerances. The uncorrected systematic errors of the iGPS system vary randomly within the measurement volume and are therefore assumed to be normal distributions rather than the rectangular distribution which would more typically be used for systematic errors. Components of uncertainty due to environmental effects are also considered such as the expansion of the concrete slab due to a variation in temperature of approximately 1 degree over a $10 \mathrm{~m}$ length, and refractive effects due to temperature gradients in the air of approximately 1 degree per metre. A complete uncertainty budget for the iGPS system is given in Table 1 (Muelaner et al. 2009). 
Table 2 : Uncertainty budget for basic iGPS system as tested at Broughton

\begin{tabular}{|c|c|c|c|c|}
\hline Component & $\begin{array}{c}\text { Value } \\
(\mu \mathrm{m})\end{array}$ & Distribution & $\begin{array}{c}\text { Standard } \\
\text { Uncertainty } \\
(\mu \mathrm{m})\end{array}$ & Contribution \\
\hline Reference Standard & 25 & Normal & 25 & $0.1 \%$ \\
\hline Repeatability of iGPS (RSS StDev) & 377 & Normal & 377 & $51 \%$ \\
\hline Rounding errors of iGPS & 0.5 & Rectangular & 0.4 & $0.00 \%$ \\
\hline Expansion of Concrete slab & 120 & Rectangular & 84.9 & $1 \%$ \\
\hline Refractive effects & 10 & Rectangular & 7.1 & $0.01 \%$ \\
\hline Uncorrected Systematic Error & 318 & Normal & 318 & $29 \%$ \\
\hline \multicolumn{3}{|c|}{ Combined Standard Uncertainty } & 503 & \\
\hline \multicolumn{3}{|c|}{ Expanded Uncertainty at $95 \%$ Confidence Level } & 1,006 & \\
\hline
\end{tabular}


Table 2 shows not only the uncertainty budget but also the contribution of each component to the combined uncertainty. This clearly shows that the majority of components have a negligible effect with only the repeatability of the iGPS system and the uncorrected systematic error making a significant contribution.

The length between each point position was also calculated and a comparison made in this way between the Laser Tracker Calibration and the iGPS measurements. The uncertainty budget was calculated in the same way for the length based measurements in the same way as for the coordinate measurements.

\section{Results}

The results presented here include both coordinate measurements (Table 3 ) and length based verification (Figure $6 \&$ Figure 7).

Table 3 : Coordinate uncertainty for tests carried out

\begin{tabular}{|c|c|c|}
\hline $\begin{array}{c}\text { Component - Standard } \\
\text { Uncertainties }\end{array}$ & Bath LIMA & Broughton \\
\hline Reference Standard $(\mu \mathrm{m})$ & 25 & 25 \\
\hline Repeatability of iGPS $(\mu \mathrm{m})$ & 289 & 377 \\
\hline $\begin{array}{c}\text { Rounding errors of iGPS } \\
(\mu \mathrm{m})\end{array}$ & 0.4 & 0.4 \\
\hline $\begin{array}{c}\text { Expansion of Concrete } \\
\text { slab }(\mu \mathrm{m})\end{array}$ & 84.9 & 84.9 \\
\hline Refractive effects $(\mu \mathrm{m})$ & 7.1 & 7.1 \\
\hline Systematic Error $(\mu \mathrm{m})$ & 505 & 318 \\
\hline $\begin{array}{c}\text { Combined Standard } \\
\text { Uncertainty }(\mu \mathrm{m})\end{array}$ & 590 & 503 \\
\hline $\begin{array}{c}\text { Expanded Uncertainty at } \\
95 \%(\mu \mathrm{m})\end{array}$ & 1,180 & 1,006 \\
\hline
\end{tabular}

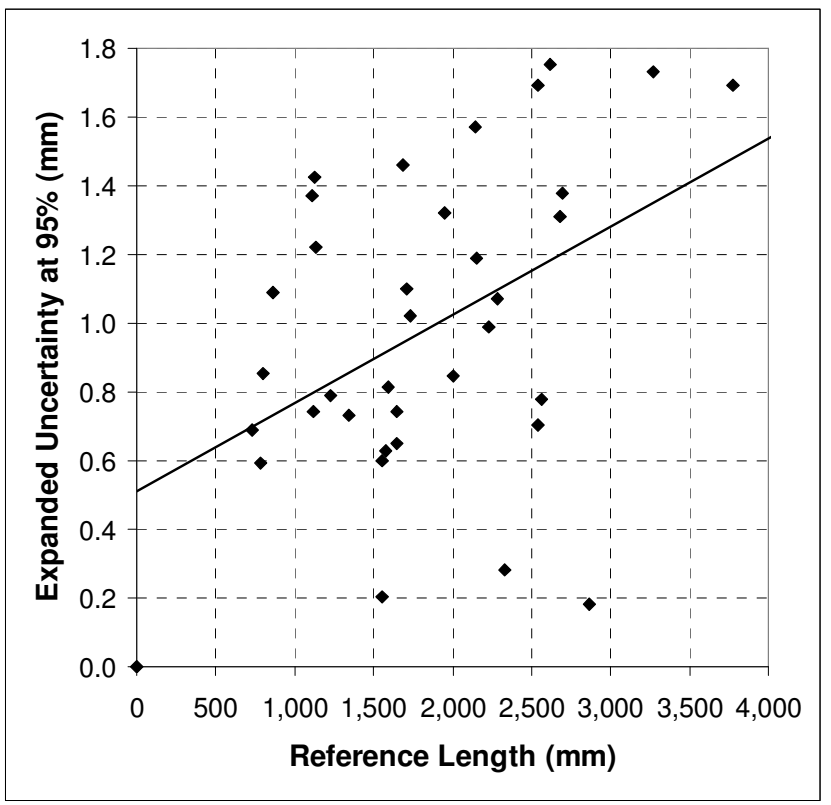

Figure 6 : Comparison of lengths for tests carried out at Bath LIMA 


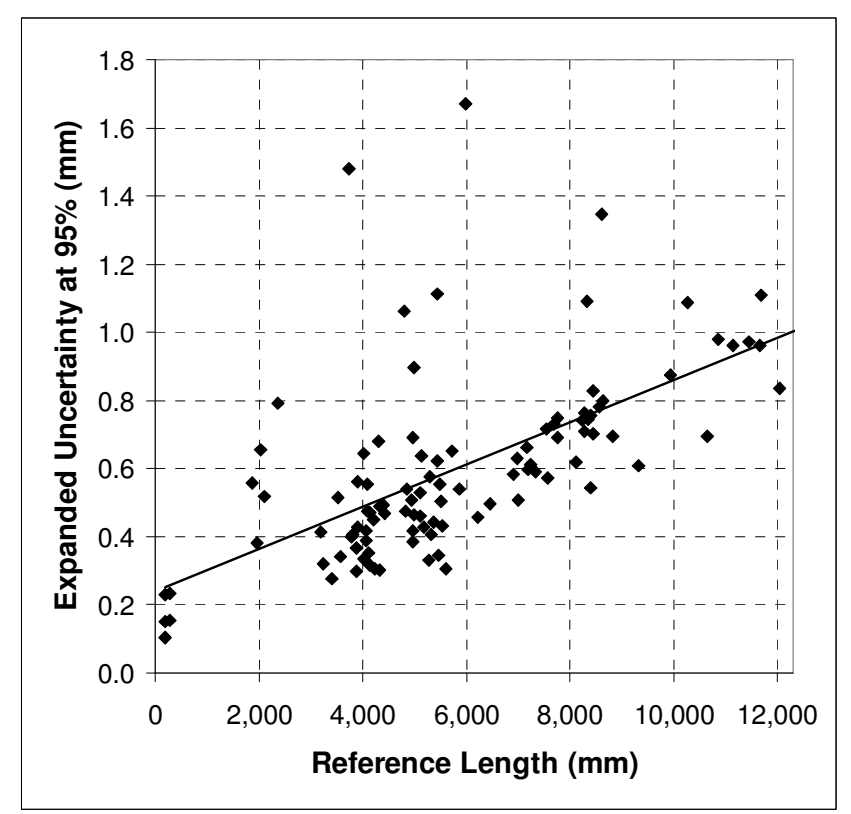

Figure 7: Comparison of lengths for tests carried out at Airbus Broughton

The performance of the system differed over the two tests with a somewhat higher uncertainty and very little evidence of length dependence in the Bath LIMA tests, and lower uncertainty with more evidence of length dependence in the Broughton tests. These differences may be explained by differences in setup procedures. The iGPS setup procedures at Broughton involved more reference lengths but this would be expected to affect the systematic error rather than variability. The expanded uncertainties calculated using length based verification were similar to the coordinate results discussed above.

The test results indicate an expanded coordinate uncertainty magnitude at a 95\% confidence level of between $+/-1 \mathrm{~mm}$ and $+/-1.1 \mathrm{~mm}$. It should be noted that these tests were carried out using a basic version of the iGPS interface software which is not the state of the art interface.

Since a weak relationship between reference length and uncertainty was seen and the systematic effects appeared to vary randomly throughout the measurement volume these figures apply throughout the measurement volume of approximately $10 \mathrm{~m} \times 10 \mathrm{~m}$ by 1.5 $\mathrm{m}$. The uncertainty interval given represents results seen in different tests.

\section{Additional Coordinate Tests}

Additional tests have been carried out on a state of the art iGPS system, which was operated and continually monitored by a team of engineers from the system manufacturer (Metris). This network consisted of 8 transmitters and 12 monument sensors which required over 100 lines of sight to be simultaneously maintained while measurements were being taken. It should be noted that although such a system might offer the best performance it is unlikely to be possible to maintain such a large number of lines of sight within most production environments.

These tests were carried out at Airbus Broughton with a volumetric coverage very similar to that of the previous tests at Broughton described above.

The results from these additional tests were analysed by the National Physical Laboratory (NPL) using specially created algorithms involving analytical, partial derivative based, solutions to the propagation of uncertainty in place of the USMN Monte-Carlo Simulation used in the previous tests. The method then uses a weighted least-squares 
fitting algorithm to compare the iGPS coordinate measurements with the reference network established by multilateration using a laser tracker.

The results showed that the iGPS system was able to determine lengths up to $12 \mathrm{~m}$ with an uncertainty of $0.17 \mathrm{~mm}$ and coordinates with an uncertainty of $0.12 \mathrm{~mm}$ in $\mathrm{x}$ and $\mathrm{y}$ and $0.19 \mathrm{~mm}$ in $\mathrm{z}$; a coordinate magnitude uncertainty of $0.25 \mathrm{~mm}$. All results are given at a 95\% confidence level (Hughes et al. 2010).

\section{Conclusions}

Using a basic version of iGPS with 4 transmitters and no monuments operated according to the user manual the test results indicate an expanded coordinate uncertainty magnitude at a $95 \%$ confidence level of between $+/-1 \mathrm{~mm}$ and $+/-1.1 \mathrm{~mm}$.

Using a state of the art system, with over 100 lines of sight between transmitters and monuments, which was operated and continually monitored by a team of engineers from the system manufacturer the expanded coordinate uncertainty magnitude at a $95 \%$ confidence level was reduced to approximately $0.25 \mathrm{~mm}$.

These two sets of results demonstrate the range of performance possible with current technology and different system configurations.

Previous work to verify the performance of an R-LAT showed that the angular uncertainty of an individual transmitter receiver pair was approximately 0.5 arc seconds at a 95\% confidence level (Muelaner, Wang et al. 2008). From basic trigonometry this is equivalent to 0.01 to $0.05 \mathrm{~mm}$ within the $5-20 \mathrm{~m}$ range. This is considerably less than the total uncertainty of the network acting as a coordinate measuring machine is shown be this work. These results indicate that there are additional sources of uncertainty inherent in the combined system. This shows the importance of combined system tests in addition to isolated tests of sub-systems.

The technique demonstrated here is appropriate for the verification of all types of coordinate measurement instrument.

Although the iGPS system has considerable potential some improvements in accuracy will be required in order to fully realize the potential of the system. Alternative systems based on ultrasound and photogrammetry currently also show potential to provide factory-wide measurement infrastructures.

\section{Acknowledgements}

The authors wish to thank E B Hughes and A B Forbes of the National Physical Laboratory for advice and support given, Mr Mike O'Sullivan and Mr Geraint Jones of Airbus UK for hosting the tests at Broughton and staff from Metris for their technical inputs.

A previous version of this paper was presented at the DET2009 conference held at the University of Hong Kong from 14-16 December 2009 and is included in the conference proceedings published by Springer.

This research has been carried out as part of the IdMRC at the Department of Mechanical Engineering of the University of Bath, under EPSRC Grants No. EP/E002617/1 and EP/E00184X/1. The participation of NPL was funded under a Joint Industry Project and the financial support of DIUS is acknowledged. 


\section{References}

Anchini, R., G. Di Leo, C. Liguori, A. Paolillo, A. Pietrosanto and G. Strazzullo (2007). Metrological verification of 3D scanners: A preliminary approach, San Jose, CA, United States, SPIE -International Society for Optical Engineering, Bellingham WA, WA 98227-0010, United States.

ASME (2006). Performance Evaluation of Laser-Based Spherical Coordinate Measurement Systems. B89.4.19.

BSI - British Standards Institution (2002). Geometrical Product Specifications (GPS) Acceptance and reverification tests for coordinate measuring machines (CMM) Part 2: CMMs used for measuring size. BS EN ISO 10360-2:2002.

BSI (1995). General Metrology - Part 3: Guide to the expression of uncertainty in measurement (GUM). PD 6461-3.

Calkins, J. M. (2002). Quantifying Coordinate Uncertainty Fields in Coupled Spatial Measurement Systems Mechanical Engineering. Blacksburg, Virginia Polytechnic Institute and State University. PhD: 226.

Estler, W. T., K. L. Edmundson, G. N. Peggs and D. H. Parker (2002). "Large-scale metrology - An update." CIRP Annals - Manufacturing Technology 51(2): 587-609.

Faro (2007). Faro Laser Tracker Xi. 2008.

Flack, D. (2001). Measurement Good Practice Guide No. 42: CMM Verification, National Physical Laboratory.

Hedges, T. M., H. Takagi, T. Pratt and M. J. Sobel (2003). Position Measurement System and Method Using Cone Math Calibration. U. S. Patent, Arc Second.

Hughes, B., A. Forbes, W. Sun, P. G. Maropoulos, J. E. Muelaner, J. Jamshidi and Z. Wang (2010), iGPS Capability Study. NPL Report. Teddington, NPL: 35.

Joshi, P., M. Imadabathuni, D. He, M. Al-Kateb and E. Bechhoefer (2009). "Application of the condition based maintenance checking system for aircrafts." Journal of Intelligent Manufacturing: 1-12.

Kapanoglu, M., M. Alikalfa, M. Ozkan, A. Yazıcı and O. Parlaktuna "A pattern-based genetic algorithm for multi-robot coverage path planning minimizing completion time." Journal of Intelligent Manufacturing: 1-11.

Lau, K., R. Hocken and W. Haight (1985). Automatic Laser Tracking Interferometer System for Robot Metrology, Interlaken, Switz.

Metris (2007). Training document iGPS \& WorkSpace.

Metris (2007). WorkSpace.

Muelaner, J. E., Z. Wang, J. Jamshidi, P. G. Maropoulos, A. R. Mileham, E. B. Hughes and A. B. Forbes (2008). "Study of the Uncertainty of Angle Measurement for a RotaryLaser Automatic Theodolite (R-LAT)." IMechE, Part B: J. Engineering Manufacture 223(B3): 217-229. 
Muelaner, J. E., Z. Wang, O. Martin, J. Jamshidi and P. G. Maropoulos (2009). "Estimation of Uncertainty in Three Dimensional Coordinate Measurement by Comparison with Calibrated Points." Measurement Science and Technology 21 () 025106: 9 pages.

New River Kinematics (2007). SpatialAnalyzer.

Richard, S. and B. Kendall (2006). Chirped coherent laser radar system and method.

Schwenke, H., M. Franke, J. Hannaford and H. Kunzmann (2005). "Error mapping of CMMs and machine tools by a single tracking interferometer." CIRP Annals Manufacturing Technology 54(1): 475-478.

Tang, F., I. You, S. Guo, M. Guo and Y. Ma (2010). "A chain-cluster based routing algorithm for wireless sensor networks." Journal of Intelligent Manufacturing: 1-9.

Triggs, B., P. Mclauchlan, R. Hartley and A. Fitzgibbon (1999). Bundle Adjustment - A Modern Synthesis. Vision Algorithms: Theory and Practice, International Workshop on Vision Algorithms. Corfu, Greece.

VDI/VDE-Gesellschaft Mess- und Automatisierungstechnik (2002). Optical 3D measuring systems - Imaging systems with point-by-point probing. VDI/VDE 2634 Part 1. 